\title{
VARIATIONS IN A UNIVERSAL DARK MATTER PROFILE FOR DWARF SPHEROIDALS
}

\author{
John R. JARDEL AND KaRL GEBHARDT \\ Department of Astronomy, University of Texas at Austin, 1 University Station C1400, Austin, TX 78712, USA; jardel@astro.as.utexas.edu \\ Received 2013 June 3; accepted 2013 August 16; published 2013 September 10
}

\begin{abstract}
Using a newly developed modeling technique, we present orbit-based dynamical models of the Carina, Draco, Fornax, Sculptor, and Sextans dwarf spheroidal (dSph) galaxies. These models calculate the dark matter profiles nonparametrically without requiring any assumptions to be made about their profile shapes. By lifting this restriction, we discover a host of dark matter profiles in the $\mathrm{dSphs}$ that are different from the typical profiles suggested by both theorists and observers. However, when we scale these profiles appropriately and plot them on a common axis, they appear to follow an approximate $r^{-1}$ power law with considerable scatter.
\end{abstract}

Key words: dark matter - galaxies: dwarf - galaxies: kinematics and dynamics - Local Group

Online-only material: color figures

\section{INTRODUCTION}

It is well known that cosmological simulations containing only collisionless dark matter produce halos that share a universal density profile $\rho_{\mathrm{DM}}(r)$ (Navarro et al. 1996b, 2010; Springel et al. 2008). At first, this universal profile was characterized by the double power-law Navarro-Frenk-White (NFW) profile (Navarro et al. 1996b) with an inner logarithmic slope $\alpha \equiv-d \log \rho_{\mathrm{DM}} / d \log r=1$. Modern dark-matter-only simulations with increasingly better resolution seem to produce profiles that, in analogy to the Sérsic function (Sersic 1968), transition smoothly from $\alpha=3$ in the outer regions to $\alpha \sim 1$ near the center (Merritt et al. 2005; Gao et al. 2008; Navarro et al. 2010). The exact form of $\rho_{\mathrm{DM}}(r)$ is still debated by theorists, but most agree that the inner slope is nonzero. Such profiles are called "cuspy" since $\rho_{\mathrm{DM}}$ increases as $r \rightarrow 0$. In contrast, observers modeling low-mass galaxies with stellar and gas dynamics often find $\alpha=0$ "cores" in the inner profiles (Burkert 1995; Persic et al. 1996; Borriello \& Salucci 2001; de Blok et al. 2001; Simon et al. 2005). This disagreement between theory and observations has become known as the core/cusp debate.

We must remember, however, that real galaxies are the products of their unique formation histories, and complex baryonic processes can reshape dark matter profiles in different ways. Whether originating from adiabatic compression (Blumenthal et al. 1986), supernovae winds (Navarro et al. 1996a), or rampressure stripping (Arraki et al. 2012), baryonic feedback has been shown to affect the dark matter profiles of galaxies by perturbing their baryons in a highly non-linear way. Since these processes differ on a galaxy-by-galaxy basis, one should not expect to observe a universal dark matter profile at $z=0$. Furthermore, given the number of different ways baryonic feedback can occur, we should not expect it to produce only cored or NFW-like profiles.

Unfortunately, it is difficult to explore the possible range of profile shapes since to construct a dynamical model one generally needs to adopt a parameterization for $\rho_{\mathrm{DM}}(r)$. This is not ideal as one is forced to assume the very thing they are hoping to measure. Clearly, methods that can measure $\rho_{\mathrm{DM}}(r)$ nonparametrically are advantageous. Non-parametric determination of the dark matter profile avoids biasing results by assuming an incorrect parameterization and it also allows more general profile types to be discovered.
To test the universal profile assumption, we apply the technique of non-parametric Schwarzschild modeling to determine $\rho_{\mathrm{DM}}(r)$ in five of the brightest dwarf spheroidal (dSph) galaxies that orbit the Milky Way as satellites. These galaxies have excellent kinematics available (Walker et al. 2009a) and have been demonstrated to be good targets for this type of modeling (Jardel et al. 2013). The dSphs as a population are some of the most dark-matter-dominated galaxies ever observed (Mateo 1998; Simon \& Geha 2007) and as such are unique test sites for theories of galaxy formation at low mass scales.

Past studies using Jeans models have had difficulty robustly measuring $\rho_{\mathrm{DM}}(r)$ in the dSphs (Walker et al. 2009b) largely due to the degeneracy between mass and velocity anisotropy inherent to these models. In addition to being fully non-parametric, our models break the degeneracy between mass and velocity anisotropy the same way traditional Schwarzschild models accomplish this (Gebhardt et al. 2000; Rix et al. 1997; van der Marel et al. 1998; Valluri et al. 2004; van den Bosch et al. 2008). In this Letter, we apply the most general models to a widely studied group of galaxies in order to measure their dark mater density profiles and test the universal profile hypothesis.

\section{DATA}

Our models use the publicly available kinematics data from Walker et al. (2009a) for Carina, Fornax, Sculptor, and Sextans. These data are individual radial velocities for member stars with repeat observations weighted and averaged. Walker et al. (2009a) assign each star a membership probability $P$ based on its position, velocity, and a proxy for its metallicity. Our analysis only includes stars for which $P>0.95$. Whenever a galaxy has high quality Hubble Space Telescope measurements of its proper motion available (Carina and Fornax; Piatek et al. 2003, 2007), we correct for the effects of perspective rotation following Appendix A of Walker et al. (2008).

As described in Jardel \& Gebhardt (2012), stars are placed on a meridional grid according to their positions and folded over the major and minor axes. To preserve any possible rotation, we switch the sign of the velocity whenever a star is flipped about the minor axis. We then group the stars into spatial bins by dividing the grid into a series of annular bins containing roughly 50-70 stars per bin. Fornax and Sculptor have a larger number of stars with measured velocities, and to exploit this 
Table 1

Properties of the Dwarf Spheroidals

\begin{tabular}{lcrrrr}
\hline \hline Galaxy & $\begin{array}{c}\text { Distance } \\
(\mathrm{kpc})\end{array}$ & $N_{\text {stars }}$ & $N_{\text {LOSVD }}$ & Ellipticity & $R_{\text {trunc }}$ \\
\hline Carina & $104^{\mathrm{a}}$ & $702^{\mathrm{f}}$ & 14 & $0.33^{\mathrm{e}}$ & 4.2 \\
Draco & $71^{\mathrm{b}}$ & $170^{\mathrm{g}, \mathrm{h}}$ & 8 & $0.29^{\mathrm{e}}$ & 3.1 \\
Fornax & $136^{\mathrm{a}}$ & $2409^{\mathrm{f}}$ & 36 & $0.30^{\mathrm{e}}$ & 13.5 \\
Sculptor & $85^{\mathrm{c}}$ & $1266^{\mathrm{f}}$ & 24 & $0.32^{\mathrm{e}}$ & 5.1 \\
Sextans & $85^{\mathrm{d}}$ & $388^{\mathrm{f}}$ & 8 & $0.35^{\mathrm{e}}$ & 5.1 \\
\hline
\end{tabular}

Notes. Summary of the data we use for our study of the dSphs. We list the distances to the dSphs we have assumed, the number of member stars with radial velocity measurements $N_{\text {stars }}$, and the number of LOSVDs these measurements are divided into $N_{\text {LOSVD }}$. We assume that the dark matter halo has the same ellipticity as the value listed for the stellar component. We also list the truncation radius $R_{\text {trunc }}$ used in our analysis.

References. ${ }^{\mathrm{a}}$ Tammann et al. (2008), ${ }^{\mathrm{b}}$ Odenkirchen et al. (2001), ${ }^{\mathrm{c}}$ Pietrzyński et al. (2008), ${ }^{\mathrm{d}}$ Lee et al. (2009), ${ }^{\mathrm{e}}$ Irwin \& Hatzidimitriou (1995), ${ }^{\mathrm{f}}$ Walker et al. (2009a), ${ }^{\mathrm{g}}$ Kleyna et al. (2002), ${ }^{\mathrm{h}}$ Jardel et al. (2013).

we subdivide the annular bins into two to three angular bins in analogy to spokes on a wheel. Table 1 presents a summary of the data we use for the dSphs.

For each spatial bin of stars, we reconstruct the full line-ofsight velocity distribution (LOSVD) from the discrete radial velocities observed. This procedure uses an adaptive kernel density estimator (Silverman 1986) and is described in more detail in Jardel et al. (2013). Uncertainties in the LOSVDs are determined through bootstrap resamplings of the data. We divide each LOSVD into 15 velocity bins which serve as the observational constraint for our models.

Also necessary for the models is the galaxy's threedimensional luminosity density profile $v(r)$. To obtain this, we start with the projected number density profile of stars $\Sigma_{*}(R)$. For Carina and Sculptor we take $\Sigma_{*}(R)$ from Walcher et al. (2003), opting to use their fitted King profile for Carina and the actual profile for Sculptor with no fit performed. We also use a King profile to describe $\Sigma_{*}(R)$ in Sextans, with the parameters taken from Irwin \& Hatzidimitriou (1995). In Fornax we use the full profile reported in Coleman et al. (2005). We then convert $\Sigma_{*}(R)$ to a surface brightness profile $\mu(R)$ by adding an arbitrary zero-point shift, in log space, and adjusting the shift until the integrated $\mu(R)$ returns a luminosity consistent with the value listed in Mateo (1998).

Next we deproject $\mu(R)$ via Abel inversion through the manner described in Gebhardt et al. (1996). For simplicity in the deprojection and subsequent modeling, we assume that each galaxy is viewed edge-on. For a thorough discussion on how uncertainties in viewing angle and geometry propagate through our models, we refer the reader to Thomas et al. (2007). Our models are axisymmetric, so we use the stellar ellipticity to determine $v$ away from the major axis.

\section{MODELS}

The non-parametric modeling technique we use is described in full detail in Jardel et al. (2013). It is based on the Schwarzschild modeling code of Gebhardt et al. (2000) updated by Thomas et al. (2004, 2005) and described in Siopis et al. (2009). We have tested our models by using kinematics generated from a Draco-sized mock dSph embedded in a larger dark matter halo with either a cored or NFW-like cuspy profile. In both cases, we are able to accurately recover the density profile from which the mock kinematics were drawn.
The fundamental principle behind Schwarzschild modeling, that of orbit superposition, was first introduced by Schwarzschild (1979). The Schwarzschild code that is the backbone of our non-parametric technique has been thoroughly tested using artificial data. It has been shown to accurately recover the mass profile and orbit structure of simple isotropic rotators (Thomas et al. 2005), $N$-body merger remnants (Thomas et al. 2007), and a mock galaxy containing a supermassive black hole (Siopis et al. 2009). The general Schwarzschild technique has also been tested with artificial data representing the binned individual velocities typically used as input for studying the dSphs (Breddels et al. 2013).

This method works by assuming a trial potential for the galaxy under study and determining all stellar orbits that are possible in that potential. Our orbit sampling scheme is described in detail in Thomas et al. (2004). The orbits are then assigned weights according to how well they match the LOSVDs and a $\chi^{2}$ value is determined, subject to a constraint of maximum entropy (Siopis et al. 2009). If $\chi^{2}$ is low, the orbits are a good fit to the kinematics and the trial potential is considered to be a good estimate for the real potential. If $\chi^{2}$ is large, the trial potential does not support orbits that can match the kinematics and a new potential is generated. Each model is required to match $v(r)$ as well to machine precision.

We construct the many trial potentials by solving Poisson's equation for a specified total density profile $\rho(r)$ along the major axis. We assume the total mass distribution has the same ellipticity as the stellar component and use this adopted ellipticity to define $\rho(r, \theta)$ away from the major axis.

Rather than parameterizing $\rho(r)$ with an unknown function and sampling its parameters, we take an altogether different approach (detailed in Jardel et al. 2013). To describe $\rho(r)$, we divide the profile into five radial points $r_{i}$, equally spaced in $\log r$. A trial $\rho(r)$ is then represented by the density $\rho_{i}$ at each point. In this way, the $\rho_{i}$ themselves are the parameters that we adjust when picking trial potentials. To sample this parameter space, we employ a similar iterative refinement scheme as discussed in Jardel et al. (2013). We also impose the same constraint that each profile must be non-increasing as a function of radius.

Since the dSphs orbit within the Milky Way's halo, the possibility exists that they are being, or have been, tidally stripped. In constructing our trial potential, we account for this by leaving the slope of $\rho(r)$ outside of our model grid a free parameter $\alpha_{\infty}$. Each model profile $\rho(r)$ is run with $\alpha_{\infty} \in\{2,3,4\}$. In this way, we treat $\alpha_{\infty}$ as a nuisance parameter and marginalize over it for the rest of our discussion. We also truncate the dSphs at the radius $R_{\text {trunc }}$ defined by the Jacobi radius given the mass of the $\mathrm{dSph}$, its Galactocentric distance, and the mass of the Milky Way (assumed to be $M_{\mathrm{MW}}=3 \times 10^{12} M_{\odot}$ and represented by an isothermal sphere). We list values for $R_{\text {trunc }}$ in Table 1 .

\subsection{Stellar Density}

After running a large number of models for each galaxy, we have a non-parametric measurement of the total density profile $\rho(r)$. In order to obtain the dark matter density profile, we must subtract the stellar density $\rho_{*}(r)$. This requires knowledge of the stellar mass-to-light ratio $M_{*} / L_{V}$ since $\rho_{*}(r)=M_{*} / L_{V} \times v(r)$, assuming that variations in $M_{*} / L_{V}$ with radius are unimportant.

To estimate $M_{*} / L_{V}$, we use photometrically derived determinations of each galaxy's stellar age $t_{\text {age }}$ and metallicity $[\mathrm{Fe} / \mathrm{H}]$ (Lianou et al. 2011). The simple stellar population 


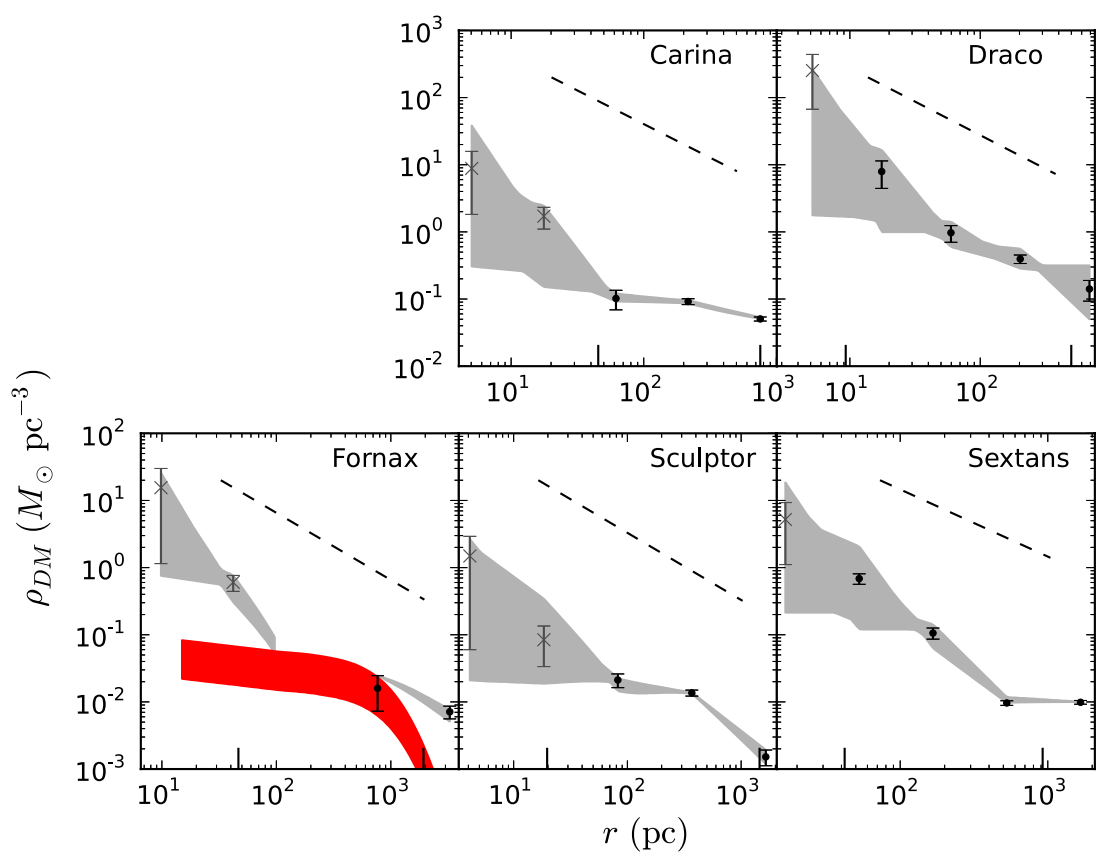

Figure 1. Dark matter density profiles for each dSph modeled as well as Draco from Jardel et al. (2013). Points with error bars show the $\Delta \chi^{2}=1$ uncertainties in the $\rho_{i}$. These points are gray X's when they lie interior to our kinematics and black dots otherwise. Vertical black tick marks on the $x$-axes show the radial extent of this range. The gray shaded regions show the joint confidence range of the entire profile at the level of $\Delta \chi^{2}=5.84$ and interpolated between the $\rho_{i}$. Each panel plots a generic $r^{-1}$ NFW-like profile as a dashed line. In Fornax we also plot the stellar density profile $\rho_{*}(r)$ with $1 \sigma$ uncertainties in red.

(A color version of this figure is available in the online journal.)

models of Maraston (2005) then yield an estimate on $M_{*} / L_{V}$ given these two quantities and the assumption of either a Salpeter or Kroupa initial mass function (IMF). We characterize the uncertainty in $\rho_{*}(r)$ by the spread in values of $M_{*} / L_{V}$ that result from a choice in IMF and the uncertainties in $t_{\text {age }}$ and $[\mathrm{Fe} / \mathrm{H}]$. For our analysis of $\rho_{\mathrm{DM}}(r)$, we add in quadrature the uncertainties on $\rho(r)$ from the models with those on $\rho_{*}$ due to $M_{*} / L_{V}$.

In all but one of the dSphs (Fornax), $\rho(r) \gg \rho_{*}(r)$ making the determination of $\rho_{*}(r)$ relatively unimportant. In Fornax, however, the relatively large uncertainties on $\rho_{*}(r)$ make $\rho(r)-\rho_{*}(r)$ a negative quantity in some cases. This is clearly unphysical as it represents a negative dark matter density. To better study Fornax and other relatively baryon-dominated galaxies, a more accurate determination of $M_{*} / L_{V}$ is required.

\section{2. $\chi^{2}$ Analysis}

We evaluate the goodness of fit of each model with $\chi^{2}$ as calculated by

$$
\chi^{2}=\sum_{i=1}^{N_{\mathrm{LOSvD}}} \sum_{j=1}^{N_{\text {vel }}=15}\left(\frac{\ell_{i j}^{\mathrm{obs}}-\ell_{i j}^{\mathrm{mod}}}{\sigma_{i j}}\right)^{2},
$$

where the sums are computed over the $N_{\text {vel }}=15$ velocity bins for all of the LOSVDs in each galaxy. The $\ell_{i j}$ correspond to the value in the $j$ th velocity bin of the $i$ th LOSVD. The uncertainty in $\ell_{i j}^{\mathrm{obs}}$ is $\sigma_{i j}$.

We identify the best-fitting model as that which has the lowest value of the (unreduced) $\chi^{2}=\chi_{\min }^{2}$. A naïve calculation of the reduced $\chi_{v}^{2}=\chi_{\min }^{2} /\left(N_{\text {vel }} \times N_{\text {LOSVD }}\right)$ often yields values much less than unity due to correlation between velocity bins caused by our kernel density estimator. We instead test for the overall goodness of fit of our best model by computing $\chi_{v, G H}^{2}$ : the reduced $\chi^{2}$ with respect to a Gauss-Hermite parameterization of our best-fitting LOSVDs. We find $\chi_{v, G H}^{2}$ ranges from 0.3 to 0.9 for the four dSphs modeled here. These values are consistent with past results (Gebhardt et al. 2003; Gebhardt \& Thomas 2009; Jardel et al. 2013) and have been demonstrated to lead to accurate recovery of the mass profiles of mock galaxies (Thomas et al. 2005). We therefore scale our model-computed unreduced $\chi^{2}$ values by a factor equal to $\chi_{v}^{2} / \chi_{v, G H}^{2}$ in order to bring our reduced $\chi_{v}^{2}$ nearer to $\chi_{v, G H}^{2}$.

We present our dark matter profiles at two different levels of confidence. When specifying the dark matter density at a single point, we marginalize over all other parameters using the sliding boxcar technique described in Jardel et al. (2013) to interpolate $\chi^{2}$. The $1 \sigma$ confidence interval thus corresponds to a limit of $\Delta \chi^{2}=1$ (for one degree of freedom) above $\chi_{\min }^{2}$. When referring to the joint $1 \sigma$ confidence interval of the entire profile, we instead include limits derived from all models within $\Delta \chi^{2}=5.84$ of $\chi_{\min }^{2}$ (for five degrees of freedom).

\section{RESULTS}

We present the non-parametrically determined dark matter profiles in Figure 1. In addition to the new results for Carina, Fornax, Sculptor, and Sextans, we include the result from Jardel et al. (2013) for Draco. Each panel in Figure 1 contains a dashed line with $\rho_{\mathrm{DM}} \propto r^{-1}$ to show the generic shape of the NFW profile. The points with error bars in Figure 1 are the marginalized dark matter density determined from $\Delta \chi^{2}=1$ at the $r_{i}$ where the total density is being varied from model to model. The gray points labeled with X's are located interior to the radial range over which stellar kinematics are available. We denote this range for each galaxy with vertical tick marks on the $x$-axis. The joint confidence band (shaded region) interpolates between the $r_{i}$ by taking the maximum and minimum value for $\rho_{\mathrm{DM}}$ at each radius for every model within $\Delta \chi^{2}=5.84$ of $\chi_{\min }^{2}$. 
Given the freedom to choose a dark matter profile of any shape, it is immediately apparent that our models have chosen a variety of shapes for the dSphs. Draco appears the most similar to the NFW profile while Sculptor most closely resembles a broken power law that becomes shallower toward its center. The other galaxies host profiles that resemble neither cores nor cusps: Carina's profile appears flat where we have kinematics but then displays a possible up-bending inside of this region. Sextans has a steeper slope than the NFW profile until its outermost point where it suddenly becomes flat. These sharp differences among $\mathrm{dSph}$ dark matter profiles demonstrate the variety of profile shapes in the Local Group.

Unfortunately, due to a lack of central stellar velocities in the Walker et al. (2009a) data, the central profiles of the dSphs we model become increasingly uncertain there. This is evidenced by the larger error bars on our gray points in Figure 1 where we have no kinematics coverage. However, we do have some constraint from projection effects and radial orbits in our models that have apocenters at radii where we do have data.

\subsection{Fornax}

Fornax is an especially difficult case for non-parametric modeling because, compared to the other dSphs, it is relatively baryon-dominated. Our imprecise determination of $M_{*} / L_{V}$ in Fornax causes $\rho_{*}(r)$ to be greater than the total modeled density at some radii, making $\rho_{\mathrm{DM}}(r)$ negative. In our analysis of Fornax, we do not plot the radial range over which this occurs as it is unphysical. Instead, in Figure 1 we overplot the stellar density in red to illustrate why the subtraction is difficult in Fornax. In all other panels, $\rho_{*}(r) \ll \rho_{\mathrm{DM}}(r)$ and is not plotted.

There is strong evidence from multiple studies using independent methods that suggests that Fornax has a dark matter profile that is not cuspy like the NFW profile. (Goerdt et al. 2006; Walker \& Peñarrubia 2011; Jardel \& Gebhardt 2012). Each of these studies only contrasts between cored and cuspy profiles or uses a single slope to characterize the profile. It is therefore interesting to explore the non-parametric result we obtain. Even though we cannot determine $\rho_{\mathrm{DM}}$ where the stellar density is greater than the total density, we can still place an upper limit on $\rho_{\text {DM }}$ such that it must not be greater than $\rho_{*}$ or the red band in Figure 1. Given this constraint, we can see that the outer profile of Fornax is flat, while the inner portion rises more steeply than $r^{-1}$. Past dynamical studies of Fornax only compared generic cored and NFW profiles and did not test this up-bending profile, therefore it is difficult to compare to their results.

\subsection{A Common Halo?}

Despite the differences in the individual profiles of the dSphs, when we plot them on the same axes they appear to follow a combined $r^{-1}$ profile with scatter. We plot this combined profile in Figure 2 with each galaxy's profile as a separate color. The uncertainties on the points are the $\Delta \chi^{2}=1$ uncertainties from Figure 1. We have scaled each galaxy's profile relative to an arbitrary $r^{-1}$ profile. In this way, the shape of each profile is preserved and only the height has been adjusted to reduce the scatter. We fit a line to the $\log \rho_{\mathrm{DM}}$ profiles and determine that the slope $\alpha=1.2 \pm 0.5$. We also restrict our fit to only points in the profile where we have kinematics (dotted line in Figure 2) and find a similar slope of $\alpha=0.9 \pm 0.5$.

We conclude from Figure 2 that the average dark matter profile in the dSphs is similar to an $r^{-1}$ profile. However, when we model each galaxy individually, we find a variety of profiles

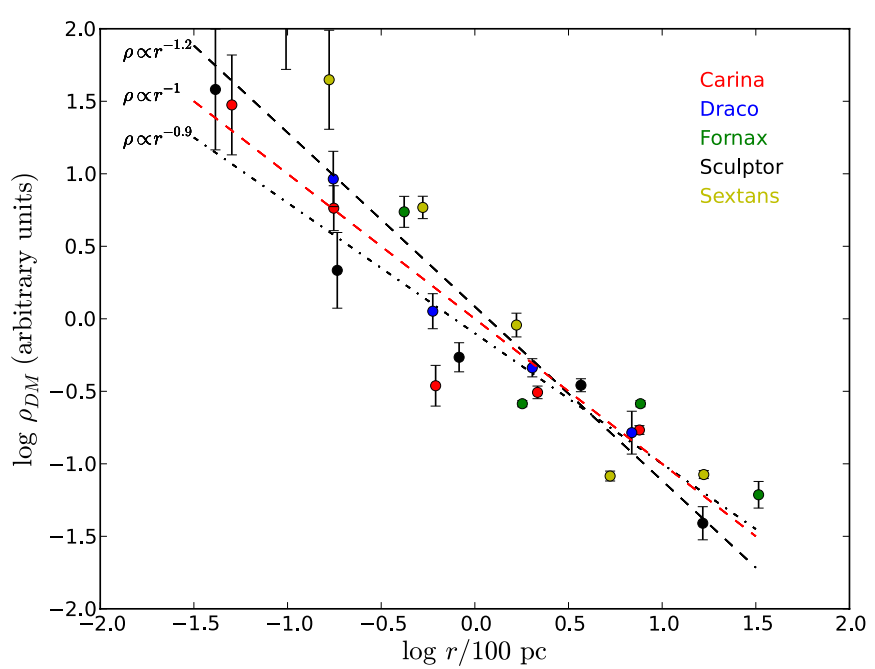

Figure 2. Combined dark matter density profiles of all the dSphs plotted on the same axes. Each galaxy's profile is plotted with the same colored points. Uncertainties on these points are the $\Delta \chi^{2}=1$ uncertainties from Figure 1. We plot the derived best-fit line with slope $\alpha=1.2 \pm 0.5$ as a dashed line as well as the NFW profile with $\alpha=1.0$ as a red dashed line. A fit excluding the points where we have no kinematics available is shown as a dotted line. The individual profiles have been scaled to a common height.

(A color version of this figure is available in the online journal.)

that are different from the mean $r^{-1}$ profile. Our interpretation of this observation is that variations in their individual formation histories cause galaxies to scatter from the average profile. Only when multiple galaxies are averaged together does it become clear they follow a combined $r^{-1}$ profile. This single powerlaw profile compares well with the predicted NFW profile in the inner portion of the plot. However, at larger radii ( $\gtrsim 1 \mathrm{kpc}$ in dwarf galaxies) the NFW profile becomes steeper than $r^{-1}$ (Springel et al. 2008). More data are needed at both large and small radii to further explore this.

K.G. acknowledges support from NSF-0908639. This work would not be possible without the state-of-the-art supercomputing facilities at the Texas Advanced Computing Center (TACC). We also thank Matt Walker and the MMFS Survey team for making their radial velocities publicly available.

\section{REFERENCES}

Arraki, K. S., Klypin, A., More, S., \& Trujillo-Gomez, S. 2012, arXiv: 1212.6651

Blumenthal, G. R., Faber, S. M., Flores, R., \& Primack, J. R. 1986, ApJ, 301,27

Borriello, A., \& Salucci, P. 2001, MNRAS, 323, 285

Breddels, M. A., Helmi, A., van den Bosch, R. C. E., van de Ven, G., \& Battaglia, G. 2013, MNRAS, 433, 3173

Burkert, A. 1995, ApJL, 447, L25

Coleman, M. G., Da Costa, G. S., Bland-Hawthorn, J., \& Freeman, K. C. 2005, AJ, 129, 1443

de Blok, W. J. G., McGaugh, S. S., Bosma, A., \& Rubin, V. C. 2001, ApJL, $552, \mathrm{~L} 23$

Gao, L., Navarro, J. F., Cole, S., et al. 2008, MNRAS, 387, 536

Gebhardt, K., Bender, R., Bower, G., et al. 2000, ApJL, 539, L13

Gebhardt, K., Richstone, D., Ajhar, E. A., et al. 1996, AJ, 112, 105

Gebhardt, K., Richstone, D., Tremaine, S., et al. 2003, ApJ, 583, 92

Gebhardt, K., \& Thomas, J. 2009, ApJ, 700, 1690

Goerdt, T., Moore, B., Read, J. I., Stadel, J., \& Zemp, M. 2006, MNRAS, 368, 1073

Irwin, M., \& Hatzidimitriou, D. 1995, MNRAS, 277, 1354

Jardel, J. R., \& Gebhardt, K. 2012, ApJ, 746, 89 
Jardel, J. R., Gebhardt, K., Fabricius, M. H., Drory, N., \& Williams, M. J. 2013, ApJ, 763, 91

Kleyna, J., Wilkinson, M. I., Evans, N. W., Gilmore, G., \& Frayn, C. 2002, MNRAS, 330, 792

Lee, M. G., Yuk, I.-S., Park, H. S., Harris, J., \& Zaritsky, D. 2009, ApJ, 703, 692

Lianou, S., Grebel, E. K., \& Koch, A. 2011, A\&A, 531, A152

Maraston, C. 2005, MNRAS, 362, 799

Mateo, M. L. 1998, ARA\&A, 36, 435

Merritt, D., Navarro, J. F., Ludlow, A., \& Jenkins, A. 2005, ApJL, 624, L85

Navarro, J. F., Eke, V. R., \& Frenk, C. S. 1996a, MNRAS, 283, L72

Navarro, J. F., Frenk, C. S., \& White, S. D. M. 1996b, ApJ, 462, 563

Navarro, J. F., Ludlow, A., Springel, V., et al. 2010, MNRAS, 402, 21

Odenkirchen, M., Grebel, E. K., Harbeck, D., et al. 2001, AJ, 122, 2538

Persic, M., Salucci, P., \& Stel, F. 1996, MNRAS, 281, 27

Piatek, S., Pryor, C., Bristow, P., et al. 2007, AJ, 133, 818

Piatek, S., Pryor, C., Olszewski, E. W., et al. 2003, AJ, 126, 2346

Pietrzyński, G., Gieren, W., Szewczyk, O., et al. 2008, AJ, 135, 1993

Rix, H., de Zeeuw, P. T., Cretton, N., van der Marel, R. P., \& Carollo, C. M. 1997, ApJ, 488, 702

Schwarzschild, M. 1979, ApJ, 232, 236

Sersic, J. L. 1968, Atlas de Galaxias Australes (Cordoba, Argentina: Observatorio Astronomico, Univ. Cordoba)
Silverman, B. W. 1986, Density Estimation for Statistics and Data Analysis (London: Chapman and Hall)

Simon, J. D., Bolatto, A. D., Leroy, A., Blitz, L., \& Gates, E. L. 2005, ApJ, 621,757

Simon, J. D., \& Geha, M. 2007, ApJ, 670, 313

Siopis, C., Gebhardt, K., Lauer, T. R., et al. 2009, ApJ, 693, 946

Springel, V., Wang, J., Vogelsberger, M., et al. 2008, MNRAS, 391, 1685

Tammann, G. A., Sandage, A., \& Reindl, B. 2008, ApJ, 679, 52

Thomas, J., Jesseit, R., Naab, T., et al. 2007, MNRAS, 381, 1672

Thomas, J., Saglia, R. P., Bender, R., et al. 2004, MNRAS, 353, 391

Thomas, J., Saglia, R. P., Bender, R., et al. 2005, MNRAS, 360, 1355

Valluri, M., Merritt, D., \& Emsellem, E. 2004, ApJ, 602, 66

van den Bosch, R. C. E., van de Ven, G., Verolme, E. K., Cappellari, M., \& de Zeeuw, P. T. 2008, MNRAS, 385, 647

van der Marel, R. P., Cretton, N., de Zeeuw, P. T., \& Rix, H. 1998, ApJ, 493, 613

Walcher, C. J., Fried, J. W., Burkert, A., \& Klessen, R. S. 2003, A\&A, 406, 847

Walker, M. G., Mateo, M., \& Olszewski, E. W. 2008, ApJL, 688, L75

Walker, M. G., Mateo, M., \& Olszewski, E. W. 2009a, AJ, 137, 3100

Walker, M. G., Mateo, M., Olszewski, E. W., et al. 2009b, ApJ, 704, 1274

Walker, M. G., \& Peñarrubia, J. 2011, ApJ, 742, 20 